\title{
Implementing the HIV "treat all" guidelines in Namibia: Patient and health provider perspectives
}

Project SOAR

Follow this and additional works at: https://knowledgecommons.popcouncil.org/departments_sbsr-hiv

Part of the Demography, Population, and Ecology Commons, Family, Life Course, and Society Commons, and the International Public Health Commons How does access to this work benefit you? Let us know!

\section{Recommended Citation}

Project SOAR. 2018. "Implementing the HIV 'treat all' guidelines in Namibia: Patient and health provider perspectives," Project SOAR Results Brief. Washington, DC: Population Council. 


\section{Implementing the HIV "Treat All" Guidelines in Namibia: Patient and Health Provider Perspectives}

In late 2015, the World Health Organization announced new treatment guidelines recommending that anyone who has tested positive for HIV should begin antiretroviral therapy (ART) as soon as possible. ${ }^{1}$ In light of this policy, the Government of Namibia included "treat all" in the 2016 National Guidelines for ART, ${ }^{2}$ and began nation-wide implementation in April 2017.

In an effort to cope with the critical shortage of medical doctors (approximately 3 doctors per 10,000 population), the Namibian government began piloting ART task shifting and service decentralization in 2011 and officially adopted task-shifting guidelines in 2015 . This process involves transferring stable ART clients from district-level hospitals to smaller community health centers, and devolving specific point-ofcare responsibilities from physicians to nurses.

This brief summarizes the perspectives of clients and providers regarding ART service delivery during the early phase of Namibia's implementation of the treat all guidelines. The brief also highlights differences in views and experiences among clients pertaining to central versus decentralized ART services.

\section{METHODS}

Between June and July 2017, we conducted exit interviews with a total of 310 ART clients aged 18 years or older from 10 facilities selected from six districts in Northern Namibia. We systematically selected 226 ART clients from six district-level hospitals (central facilities) based on daily client volume. At four community-based health centers

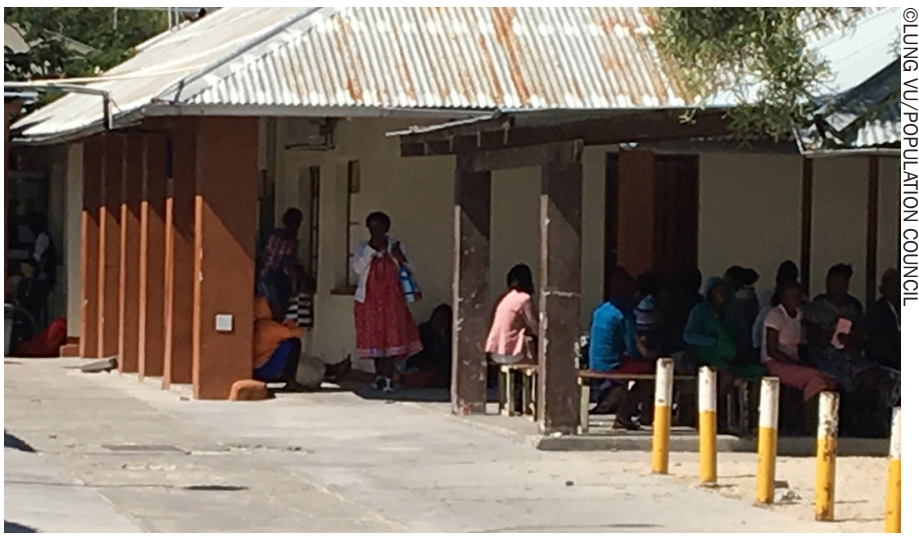

Study findings suggest that clients perceive ART service quality at decentralized, community-based facilities to be comparable to care provided at central hospitals.

\section{KEY MESSAGES}

- Data from selected ART sites show that ART clients are satisfied with the quality of care they are receiving, and perceived service quality is comparable between central and decentralized health facilities.

- Clients of both central and decentralized facilities frequently experience long waits for services; however wait times are shorter at decentralized facilities.

- Providers and clients recommended increasing the time between drug refills for stable clients to reduce client loads in facilities and alleviate service bottlenecks. 
(decentralized facilities), we interviewed all 84 ART clients. At each facility, we collected data over a one-week period. We also conducted 25 indepth interviews (IDIs) with ART clients across the 10 ART facilities and 6 focus group discussions (FGDs) with ART providers. Five FGDs were held at central facilities and one was held at a decentralized health center.

The data were collected as part of a broader assessment of how Namibia's national rollout of treat all recommendations are influencing HIV treatment outcomes. The 10 study facilities have received technical assistance for HIV clinical services from IntraHealth International (UTAP Project) since 2015.

\section{WHO PARTICIPATED IN THE EXIT INTERVIEWS?}

Nearly two-thirds were women.
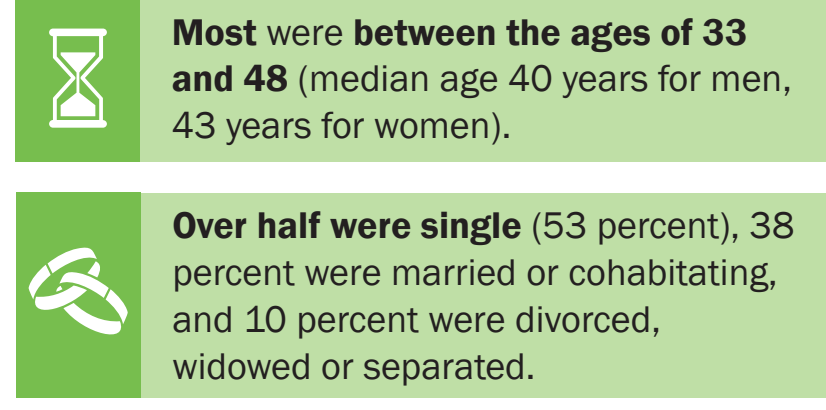

Clients had been enrolled in ART
services for an average of 6.5 years.

\section{RESULTS}

\section{Overall, clients were satisfied with ART services from either central or decentralized facilities.}

Nearly all respondents agreed they could get medical care whenever they needed it (95 percent), were treated respectfully (95 percent), and that providers spent enough time with them (87 percent).

During the IDIs many clients mentioned being satisfied with their providers because they were both kind and knowledgeable.

She treated me very nice.... She was able to answer the questions that I asked her.

-Male ART client, 23 years old, central facility

Clients also appreciated the importance of visiting the facility, as receiving medication is essential to successful treatment.

[What I like about this facility is that] it keeps me healthy.

$$
\begin{array}{r}
\text {-Female ART client, } 24 \text { years old, } \\
\text { decentralized facility }
\end{array}
$$

Despite the overall high ratings of ART services, some exit interview respondents expressed dissatisfaction with several aspects of services (Figure 1), which differed significantly between central and decentralized facilities. Clients of central hospitals were over twice as likely to agree that their care provider was too business-like or impersonal, or that the client had doubts about their provider's abilities. Conversely, more clients at rural health centers felt that their providers ignored them. Information gleaned through IDIs supported these perceptions.

Sometimes the nurse or the doctor, if you complain about pain, they will look. But I do not know if the doctor is always treating me well. But it is just sometimes how I feel. But maybe they are listening to me. I do not know.

\section{-Female ART client, 47 years old,} central facility

2 | Implementing the HIV "treat all" guidelines in Namibia: patient and health provider perspectives 
Figure 1 Differences in clients' perceptions of health providers by type of facility $(n=310)$

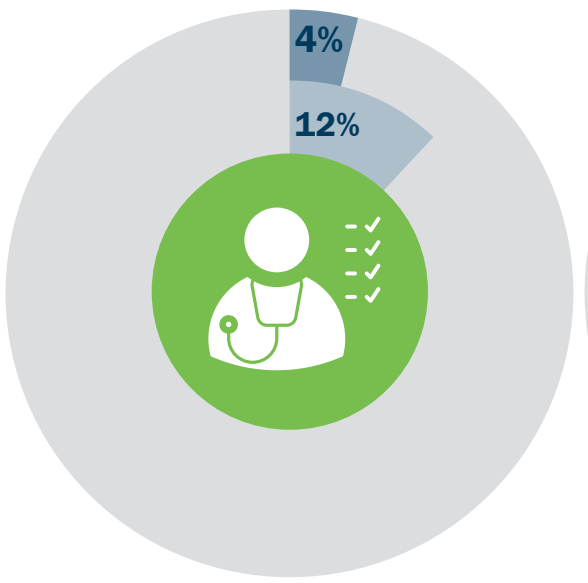

I have doubts about the abilities of the doctors who treat me*

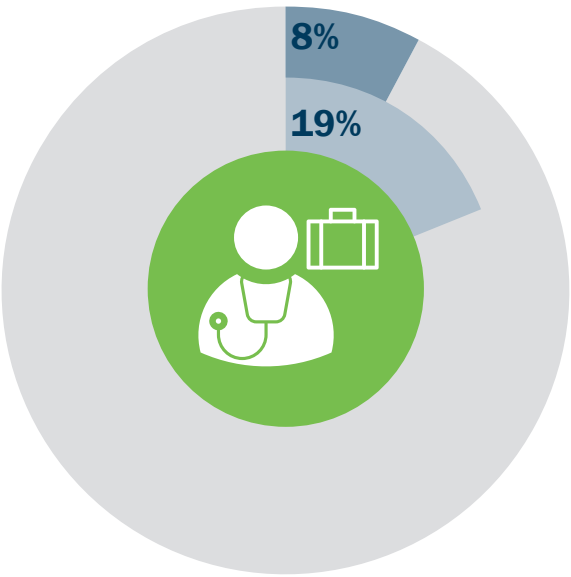

Doctors act too business-like and impersonal toward me*

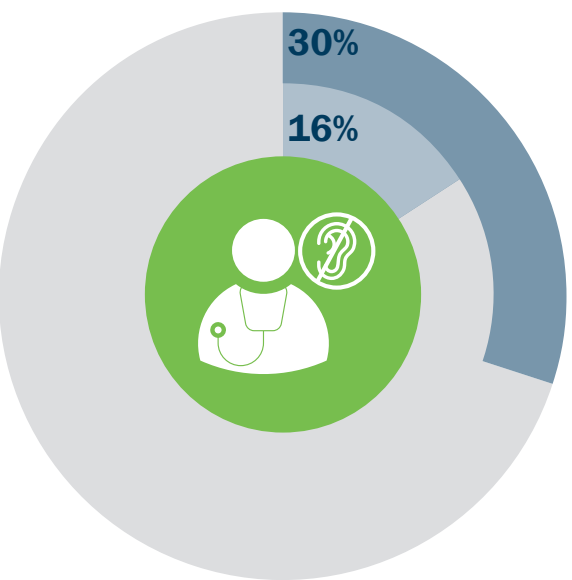

Doctors sometimes ignore what I tell them*

Centralized

Decentralized

$* p<0.05$

The rollout of the treat all guidelines has started to increase client loads.

Providers at clinics that had begun implementing the treat all guidelines indicate they are experiencing an increased number of clients, which increases workloads.

We have to enter the patients into the system-especially the ones that are starting. We have a number of new patients starting every day, especially since the new programme has started-treat all.

-Pharmacist, central facility

I would say there is a bit more workload since the implementation of this policy.

-Pharmacist assistant, central facility

\section{Structural vulnerabilities within health facilities could impede expanded ART service delivery.}

Although existing ART services provide a solid foundation for the rollout of treat all, increased workloads and client numbers could exacerbate existing weaknesses in the health system.
Overworked staff and insufficient training ART clients and clinic staff indicated that the facilities were already overburdened and understaffed, raising concerns about the increased workload treat all would bring.

The services are very good [but] we need another nurse, or even a volunteer to assist the nurse so that they can work faster.

-Female ART client, 33 years old, decentralized facility

The staff is always a challenge here.

Some of our staff go out on outreach.

Others remain at the center. If you are understaffed, you will not manage, or you will give the clients a poor service. And then you do not spend enough time with a client.

-ART nurse, central facility

Clinic staff also noted job satisfaction issues that could hinder the rollout of treat all. Clinic staff felt there were already not enough benefits to satisfy staff in the facilities, including working overtime without compensation. 
Even the staff here, they do not want to come here. They know they will work overtime, but they will not get paid for it.

-Monitoring and evaluation officer, central facility

Many clinic staff felt there needed to be better ART-related training in their facilities to establish a good foundation of knowledge and skills.

And guidelines....we need the booklets with information to guide us so that we can deliver good health services. People need to be trained and updated with the new guidelines. You cannot use the old information. Sometimes we want to send our staff to workshops [for training], but we do not always have funding.

-ART nurse, central facility

Overcrowding, staff shortages, and wait times For ART clients, overcrowding was a frequently mentioned frustration. Staff were also unhappy with the size of the facility, especially the waiting room, as they felt it did not appropriately accommodate the clients who came to the clinic.

The area here is small...the building should be made bigger. There are many people who come here to receive their medication.

-Female ART client, 33 years old, decentralized facility

To improve our service, we need to expand our space [...] the number [of patients] is still increasing on a daily basis. So we need enough space.

-Pharmacist, central facility

Staffing shortages frequently exacerbate overcrowding and contribute to long wait times during visits. This can lead to treatment default, an issue frequently mentioned by both clients and staff.
It is not short, you have to wait one or two hours. But it depends on what time you come. Even if you come here first and you are number one, the doctor can also have stuff that he has to do.

-Female ART client, 47 years old, central facility

\section{BOX 1 Transportation}

Many clients and staff noted that monetary costs, distance-related costs, and the mode of transportation available to get to the clinic impedes clients from keeping appointments. Reported transport times ranged from five minutes to four hours, each way. And, although clients of either facility type spent approximately 70 minutes traveling to the clinic, their modes of transport differed considerably. Most clients at central hospitals (56 percent) used public transport, motorcycle taxis, or private-hire cars to attend services, whereas a similar proportion (58 percent) of clients at decentralized health centers walked to the clinic.

Access to services is a problem. You find that people want to come. But then they live far away. And they have transport issues. $N \$ 10$ or $N \$ 7$ for a taxi.... We might think it is not a lot. But for some, it is not easy to get this.

$$
\text { -ART nurse, central facility }
$$

It is difficult [to come to follow-up visits]. He gives me one month. The problem is just money. When you have to pay the taxi, it is $N \$ 30$ to come and $N \$ 30$ to go back. It is a lot.

-Female ART client, 40 years old, central facility 
And when the center is overcrowded, some people will not come. Just because they have to wait so long in the queue.

$$
\text { -ART nurse, central facility }
$$

Based on data from the exit interviews, clients at decentralized facilities reported shorter median wait times than did clients at central sites (Figure 2). Overall, median wait times were shorter at decentralized sites, particularly when clients were waiting for a provider or at the pharmacy. Over a quarter of clients at central sites, and 20 percent of clients at decentralized sites reported total wait times of three hours or more.

\section{Supply management}

Clinic staff expressed concern about limited supplies of ART medication and HIV testing kits.

And when it comes to pharmaceutical issues, something I see [that] we are not doing well countrywide.... For some medicines, we are not running out, but we do not have enough [extra medication, just in case]. We should do something about that.
You have to have the HIV test kits... when it comes to the test kits we must use, they do not have them in supply. They say it is in the pipeline but it is not enough.

-Pharmacist, central facility

\section{Participants recommended expanding community ART programs and increasing the time between drug refills.}

Some central hospitals have begun implementing community-based ART programs (CbART), where nurses and community health workers provide ART outreach services to underserved rural communities. Staff in these facilities noted reductions in overall client load, allowing more time for HIV testing, counseling, and treatment of new clients.

When we started with [community] outreach, the number of clients in the center decreased. It is good...and when we provide healthcare services in the community, it has improved adherence. People no longer complain about transport money.

-Nurse, central facility

-Pharmacist assistant, central facility

Figure 2 Median wait times for services, by facility type (minutes)

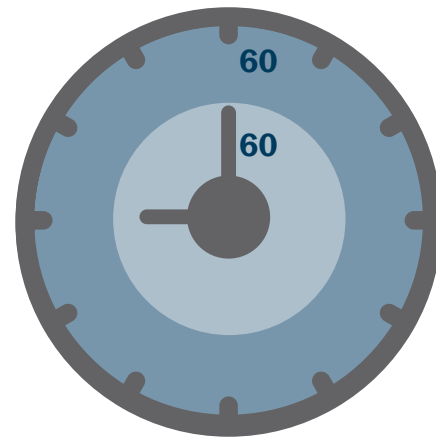

Receptionist

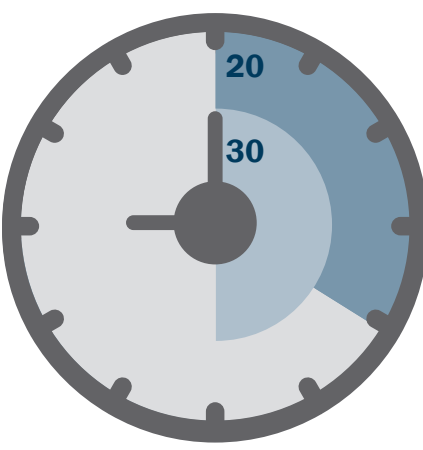

Provider*

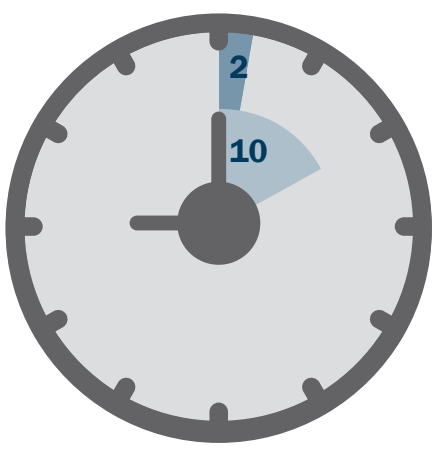

Laboratory

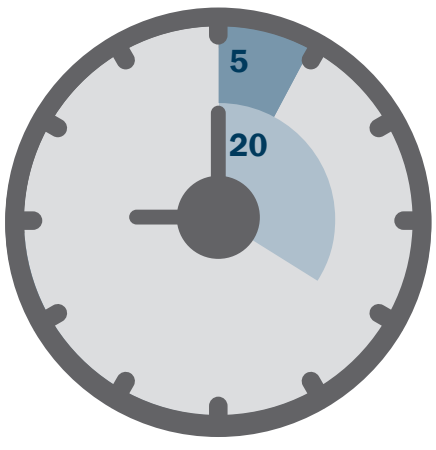

Pharmacist**

\section{Centralized}

\section{Decentralized}

$* p<0.05 ; * * p<0.0001$ 


\section{BOX 2 Other staff concerns for patients}

Many staff members were concerned that the new treat all guidelines call for patients to start treatment right away. They felt that some patients may need time to process their diagnosis before accepting the need for life-long ART. Offering ART too soon may impede their retention in care and ART adherence.

But I have some reservations about [treat all].... Expecting someone to start on the same day-that patient must be ready to start, [but] it might be too much on the patient. I do not think that it sits well with me in some cases.... I think those people must even be given a few days to think about starting this life-long treatment.

-Pharmacist, central facility
Some clients recommended extending the time between drug refill visits by increasing the amount of medication dispensed during each visit. Ideally, doing so would reduce the number of clients that visited the facility each month and reduce client default rates.

I think they give me three months [until my next follow-up].... I want it to be six months. I am busy and it is far to come to the hospital.

Male ART client, 29 years old, central facility

I come a lot of times [per year] because sometimes they give you about two months until your next follow up. And sometimes it is just a month. But it just depends on your health.... I should not come every month. A month is too short.

-Female ART client, 28 years old, central facility
One participant noted that the distance, in combination with the frequency of follow-up appointments, made it difficult to adhere to ART appointments and consequentially to adhere to ART medication.

You cannot stop this medicine. You have to come. The only problem is that they only give us [enough medication] for a month. Sometimes I have to travel far to come here. -Male ART client, 29 years old, central facility

\section{RECOMMENDATIONS}

Our findings suggest that clients perceive ART service quality at decentralized, communitybased facilities to be comparable to care provided at central hospitals. In fact, clients rated decentralized facilities higher than central facilities in some aspects of service quality, and wait times-particularly in decentralized facility pharmacies-were somewhat shorter. However, we documented a range of barriers that may impede the effective roll out of treat all in both types of facilities in Namibia. We recommend the following actions to proactively address these barriers.

\section{- Expand implementation of community-level ART services.}

Findings suggest that clients are generally satisfied with the quality of care provided through decentralized, rural health centers. This model could be expanded to improve access for clients who live far from district hospitals.

- Conduct a rigorous evaluation of CbART. Initial reports from providers suggest that community-level, outreach-based ART services can reduce patient loads at the facilities, and mitigate transport-related burdens for patients. Although further research is necessary, CbART may be a promising intervention to expand facility capacity in the treat all era.

\section{- Provide extended drug supplies to stable ART clients.}

Many clients requested that the time between drug refill appointments be increased; doing so would reduce transport burdens and opportunity costs for ART clients, and overcrowding at facilities. 


\section{- Review HIV and ART counseling guidelines to facilitate rapid ART initiation.}

Counseling messages must comprehensively address the support needs that new ART clients have, and ensure that they are prepared to adhere to lifelong ART shortly after their HIVpositive diagnosis.

- Expand human resources for health. Decrease staff stress and improve job satisfaction by hiring additional staff, enhancing training, and providing appropriate compensation and benefits.

\section{- Continue monitoring client outcomes during} the treat all rollout.

While expanding ART services to all people living with HIV is important, careful evaluation of this new approach will be critical for ensuring the country's ability to adequately respond to problems that arise which can compromise service quality.

\section{REFERENCES}

${ }^{1}$ World Health Organization. 2015. Guideline on when to start antiretroviral therapy and on preexposure prophylaxis for HIV. Geneva, Switzerland: WHO Press.

${ }^{2}$ Ministry of Health and Human Services. 2016. National Guidelines for Antiretroviral Therapy, 5th Edition. MoHSS, Windhoek.

Suggested citation: Project SOAR. 2018. "Implementing the HIV "treat all" guidelines in Namibia: patient and health provider perspectives," Project SOAR Results Brief. Washington, DC: Population Council. 
Project SOAR is a five-year (September 2014-September 2019) cooperative agreement funded by the President's Emergency Plan for AIDS Relief and the U. S. Agency for International Development (Agreement No. AIDOAA-A-14-00060). SOAR is able to accept funding from all USAID accounts.

Population Council leads the Project SOAR consortium in collaboration with Avenir Health, Elizabeth Glaser Pediatric AIDS Foundation, the Johns Hopkins University, Palladium, and The University of North Carolina at Chapel Hil.
Project SOAR/Population Council

4301 Connecticut Avenue, NW, Suite 280 Washington, DC 20008

Tel: +12022379400

e-mail: ProjectSOAR@popcouncil.org projsoar.org

(C) Population Council, March 2018 